AN. MED. INTERNA (Madrid) Vol. 18, N. ${ }^{\circ} 8$, pp. 429-431, 2001

\title{
Enfermedad de Kikuchi-Fujimoto y su asociación a lupus eritematoso sistémico
}

\author{
J. Ma . JIMÉNEZ SÁENZ, E. Mª LLORENTE ARENAS*, F. FUENTES SOLSONA, \\ F. DE MIGUEL GARCÍA*, R. ÁLVAREZ ALEGRET*** \\ Servicio de Medicina Interna. *Otorrinolaringología. **Anatomía Patológica. Hospital \\ Universitario Miguel Servet. Zaragoza.
}

\begin{abstract}
KIKUCHI-FUJIMOTO'S DISEASE AND ITS ASSOCIATION WITH SYSTEMIC LUPUS ERYTEMATOSUS
\end{abstract}

\begin{abstract}
RESUMEN
La enfermedad de Kikuchi-Fujimoto (EKF) o linfadenitis necrotizante histiocítica es una patología autolimitada que se caracteriza por linfadenopatías, fiebre y neutropenia entre otros síntomas. La etiología de la EKF es controvertida y será el estudio histológico el que proporcione el diagnóstico de confirmación.

Aunque la EKF no es muy frecuente, se han publicado varios casos en los cuales se asocia a Lupus Eritematoso Sistémico (LES) lo cual se ha de tener presente, puesto que modifica tanto el pronostico como el manejo terapéutico de estos pacientes.

Se presentan dos casos de enfermedad de Kikuchi: el primero de los casos presente una evolución clásica de la enfermedad, mientras que el segundo caso asoció un LES.
\end{abstract}

PALABRAS CLAVE: Enfermedad de Kikuchi-Fujimoto. Diagnóstico. LES.

\begin{abstract}
Kikuchi-Fujimoto's disease (KFD), Histiocytic Necrotizing lymphadenitis, is a rare self-limiting condition characterized by lymphadeno pathy, fever and neutropenia. The aetiology of KFD is controversial, and its diagnosis is confirmed histologically. Although KFD has rarely been reporded associated to Systemic lupus erythemotosus (SLE) should be ruled out given its different prognosis and management.

We present the clinical, histological and evolution the two cases of patients with Kikuchi's disease; one case had evolution classic and the other case were associated with SLE.
\end{abstract}

KEY WORDS: Kikuchi-Fujimoto's disease. Diagnostic. SLE.

Jiménez Sáenz JM", Llorente Arenas EM ${ }^{a}$, Fuentes Solsona F, de Miguel García F, Álvarez Alegret R. Enfermedad de Kikuchi-Fujimoto y su asociación a lupus eritematoso sistémico. An Med Interna (Madrid) 2001; 18: 429-431.

\section{INTRODUCCIÓN}

La enfermedad de Kikuchi-Fujimoto (EKF) fue descrita inicialmente en Japón como una linfadenitis histiocítica necrotizante subaguda en 1972 (1,2). Durante los últimos años la EKF se ha revelado como una causa creciente de linfadenopatia en Europa y Estados Unidos $(3,4)$.

La etiopatogenia de dicha enfermedad es desconocida, estando implicados varios factores víricos y/o autoinmunes $(4,5)$.

Afecta más frecuentemente a mujeres en la tercera década de la vida (6).

Desde el punto de vista clínico la EKF suele presentarse en forma de adenopatias cervicales, asociado con síntomas generales como fiebre y síntomas constitucionales $(3,4,7)$.

El diagnóstico se realiza a través del estudio anatomopatológico (8-10).

$\mathrm{Su}$ curso es benigno y autolimitado con tendencia a la remisión espontánea en 2 ó 3 meses (9), aunque se han descri- to casos recurrentes (11) así como al desarrollo de Lupus Eritematoso Sistémico (LES) en su evolución $(12,13)$.

Presentamos dos nuevos casos de EFK uno de los cuales presentó una evolución clásica de la enfermedad, mientras que el otro asoció un LES. Tras contrastar nuestros datos con los de la literatura, creemos oportuno la necesidad de hacer un seguimiento evolutivo en estos pacientes para poder establecer si presentan un evolución clásica autolimitada o por el contrario asocian algún tipo de cuadro autoinmune como el LES, en cuyo caso el pronóstico y manejo terapéutico será diferente.

\section{CASOS APORTADOS}

Caso 1: Mujer de 23 años de edad, estudiante, con antecedentes de psoriasis, que refiere desde hace dos semanas fiebre de $39^{\circ} \mathrm{C}$, de predominio nocturno, acompañado de sudoración profusa, astenia, pérdida de $5 \mathrm{Kg}$ de peso y aparición de lesiones nodulares múltiples

Trabajo aceptado: 29 de Noviembre de 1999

Correspondencia: J. Mª Jiménez Sáenz. Alfonso Zapater Cerdán, 2, 4ºB. 50014 Zaragoza. 
de crecimiento progresivo en región laterocervical izquierda. A la exploración presentaba conglomerado adenopático en cadena laterocervical izquierda de $5 \mathrm{~cm}$, de consistencia blanda, dolorosas y adheridas. Asimismo se palpaban adenopatias supraclaviculares bilaterales de $1 \mathrm{~cm}$ de diámetro, no dolorosas y desplazables.

Las pruebas complementarias revelaron: leucocitos $3.300 / \mathrm{ml}^{3}$ (54\% granulocitos, 32\% linfocitos, 14\% monocitos), plaquetas 105.000. VSG $72 \mathrm{~mm}$ la primera hora. En el proteinograma se cuantificó una fracción gammaglobulina del $28 \%$. ANA- negativo. El resto de las pruebas bioquímicas fueron normales.

La serología para brucella, toxoplasma, Epstein-Barr, CMV, lúes, VIH y Leishmania por inmunofluorescencia fueron negativas.

En la TAC cervical tras la inyección IV de contraste, se puso de manifiesto la presencia de múltiples adenopatías cervicales izquierdas con captación de contraste periférico en forma arrosariada por debajo del esternocleidomastoideo y que englobaban la cadena yugulocarotidea homolateral.

La citología realizada mediante PAAF no fue concluyente desde el punto de vista microbiológico y citológico, por lo que se realizo biopsia excisional de una adenopatía (Fig. 1), que el Servicio de Anatomía Patológica informo como linfadenitis histiciocitaria necrotizante de Kikuchi-Fujimoto.

La evolución fue favorable autolimitándose los síntomas en 2 meses. Tras la revisión del paciente de forma ambulatoria a los 6 meses se confirma la ausencia de síntomas en relación con la enfermedad.

Caso 2: Mujer de 30 años sin antecedentes de interés que presenta fiebre de $39^{\circ} \mathrm{C}$ de 10 días de evolución con tos no productiva y molestias abdominales. La palpación cervical demostró la existencia de adenopatias laterocervicales y axilares de $1 \mathrm{~cm}$ diámetro. A la exploración abdominal presentaba dolor difuso a la palpación superficial, con peristaltismo discretamente aumentado sin signos de irritación peritoneal ni visceromegalias.

En la analítica presentaba: leucocitos $3.470 / \mathrm{mm}^{3}$ con $70 \%$ de neutrofilos, serie roja y plaquetas normales. VSG $98 \mathrm{~mm}$ a la primera hora, GOT 880 UI,GPT 548 UI, PA 329 UI, LDH 948 UI, proteínas totales $6,8 \mathrm{grs} / \mathrm{dl}$ (ganmaglobulinas 34,5\%), y discreta elevación de Ig G, Ig E, Ig A.

Cultivos seriados y baciloscopias en distintas muestras biológicas resultaron negativas.

Serología para CMV 1/52, virus de Epstein-Barr, HVC, HVB, yersinia, salmonella y toxoplasma negativos.

Las pruebas de imagen mostraron hepatoesplenomegalia y adenopatias abdominales, iliacas, inguinales, axilares y laterocervicales. La biopsia de una adenopatia cervical fue diagnosticada de EKF.

La evolución fue tórpida con fiebre en picos y deterioro progresivo del estado general, en contra del diagnóstico y a pesar de varios tratamientos antibióticos empíricos. Progresivamente aparecieron artralgias, lesiones eritematopapulosas en tórax y extremidades superiores, cuadro de dolor abdominal con intolerancia alimenticia. Analíticamente presentó datos de anemia severa, leucopenia títulos positivos de ANA con patrón homogéneo, antiDNA, anti-RNP y anti-scl 70.

Tras la confirmación del diagnóstico de LES se inició tratamiento con cortisona a dosis de $2 \mathrm{mg} / \mathrm{kg} /$ día con reducción progresiva hasta completar 2 meses de tratamiento y manteniéndose una dosis de $15 \mathrm{mg} /$ día.

La paciente presentó mejoría clínica con recaída a los 6 meses del tratamiento coincidiendo con la reducción corticoidea.

\section{DISCUSIÓN}

La enfermedad de Kikuchi-Fujimoto debe considerarse como una rara causa de linfadenopatía benigna, inicialmente descrita en Japón en la década de los setenta. Aunque su inci- dencia no esta aún establecida, existen estudios que indican que pudiera ser más alta de lo que se supone (14).

Suele afectar con mayor frecuencia a mujeres en una proporción de 5:1, siendo más frecuente su presentación en pacientes con unos rangos de edad entre 11 y 75 años (4).

La etiología de la EFK no está determinada, sin embargo, los hallazgos clínicos e histológicos hacen referencia a infecciones vírales: virus de Epstein-Barr (VEB), virus parainfluenza y herpes virus $6(4,5,15)$.

Otros autores $(3,16,17)$ consideran que la ausencia de procesos faríngeos infecciosos, los cultivos negativos y la no respuesta a antibióticos indican que se trata de un proceso no bacteriano y que la existencia de células linfocitarias e histiocíticas hablarían en favor de una linfadenitis hiperinmune reactiva a diversos factores infecciosos, irritativos, tóxicos e incluso neoplásicos.

Clínicamente se caracteriza por adenopatias que suelen localizarse en región cervical (75\% de los casos) con preferencia por el triángulo posterior del cuello y con menos frecuencia en región supraclavicular, retroperitoneal e inguinal y habitualmente hepatoesplenomegalia. Suele asociar fiebre moderada, pérdida de peso, náuseas, astenia y alteraciones analíticas diversas (leucopenia a expensas de granulocitos y VSG superior a 60) (4).

El 30\% de los pacientes presentan manifestaciones cutáneas, las cuales pueden ser polimorfas (18).

Actualmente, y en ello coinciden todos los autores consultados, no existen técnicas diagnósticas no invasivas para diagnosticar este proceso, por lo tanto, la biopsia excisional y su estudio anatomopatológico determinan el diagnóstico $(3,4,19)$.

La punción aspiración con agujas fina (PAAF) puede no ser específica en estos casos, aunque puede excluir otros procesos (20).

La EKF viene definida histológicamente por: a) áreas circunscritas de células eosinofílicas con procesos necróticos; b) abundantes cariorrescis con detritus nucleares en las zonas de necrósis; c) ausencia de granulocitos y pocas células plasmáticas; d) abundantes linfocitos transformados (inmunoblastos) de origen $\mathrm{T}$ y e) presencia de monocitos plasmocitoides (2123).

Puesto que las adenopatías laterocervicales suelen ser la primera manifestación de está enfermedad, obliga a realizar diagnóstico diferencial con linfadenitis necrotizante tipo LES, procesos bacterianos y proliferaciones malignas de tipo histiocítico (LNH) (10).

Hay autores que dicen que los hallazgos anátomo-patológicos entre LES y EKF son indistinguibles (4), aunque infrecuentemente podemos encontrar cuerpos hematoxilínicos que serían un hecho anátomo-patológico diferencial entre ambas entidades (24).

Aunque la evolución habitual de la enfermedad es hacia la resolución espontánea en prácticamente todos los pacientes, en algunos de ellos se ha constatado la aparición de procesos autoinmunes concretamente LES, el cual puede preceder, ser concomitante o posterior al diagnóstico de EKF, razón por la cual se recomienda un seguimiento posterior del paciente. (25)

Tras la confirmación del diagnóstico de EKF con una evolución clásica el tratamiento es sintomático sin haberse demostrado cambios en la evolución con la administración de antibióticos y/o corticoides, siendo el proceso autolimitado en la mayoría de los casos.

Cuando la EKF presente una evolución asociada a LES, el 
cuadro ya no es autolimitado, debiendo administrase la terapéutica adecuada y revisiones periódicas que son las que darán el pronóstico evolutivo en cada paciente.

En conclusión, la EKF representa una entidad clínicopatológica bien definida, de etiopatogenia todavía no aclarada, de curso clínico benigno, habitualmente autolimitada, y con una histopatología peculiar y característica. A pesar de su rareza, su diagnóstico debe ser considerado ante situaciones clínicas de linfadenopatia localizada o generalizada no explicada y que debido a que en ocasiones asocia cuadros autoinmunes tipo LES, podría aproximarse más a un síndrome que a una enfermedad aislada.

\section{Bibliografía}

1. Kikuchi M. Lymphadenitis showing focal reticulum cell hyperplasia with nuclear debris and phagocytes: a clinicopathological study Acta Hematol Jpn. 1972; 35: 379-80.

2. Fujimoto Y, Kojima Y, Yamaguchi K. Cervical subacute necrtotizing lymphadenitis. Naika 1972; 30: 920-7.

3. Turner RR, Martin J, Dorfman RF. Necrotizing lymphadenitis, a study of 30 cases. Am J Surg Pathol 1983; 7: 115-23.

4. Dorfman RF, Berry GJ. Kikuchi's histiocytic necrotizing lymphadenitis. An analysis of 108 cases with emphasis on differential diagnosis Semin Diagno Pathol 1988; 5: 329-45.

5. Hollingsworth HC, Peiper SC, Weiss LM, Raffeld M, Jaffe ES. An investigation of the viral pathogenesis of Kikuchi-Fujimoto's disease. Lack of evidence for Epstein-Barr virus or human herpes virus 6 as the causative agents. Arch Pathol Lab Med 1994; 118: 134-40.

6. Sumiyoshi Y, Kikuchi M, Takeshita M et al. Inmunohistologic studies of Kikuchi's disease. Hum Pathol 193; 24: 1114-9.

7. Tsang WYW, Chan JKC, Mg CS. Kikuchi's lymphadenitis: a morphologic abalysis of 75 cases with special reference to unusual features. Am J Surg Pathol 1994; 18: 219-31.

8. Zabaleta M, Mollá FJ, Martínez MI, Sánchez L, Erdozain I. Enfermedad de Kikuchi. Acta Otorrinolaringol. Esp 1997; 48(4): 322-4.

9. Kikuchi M. Histiocityc necrotizing lymphadenitis (Kikuchi-Fujimoto disease) in Japan Am J Surg Pathol 1991; 197-8

10. Snow RL, Ferry JA. A 24-year old woman with cervical lymphadenopathy, fever and leukopenia. N Engl J Med 1997; 336: 492-99.

11. Kuo TT. Kikuchi's diseases. A clinocopathologic study of 79 cases with an analysis of histologic subtypes, inmunohistology, and DNA ploidy Am J Surg Pathol 1995; 19: 798-809.

12. El-Ramahi KM, Karrar A, Ali MA. Kikuchi's disease and its assiociation with systemic lupus erythematosus. Lupus 1994; 3: 409-11.

13. Litwin MD, Kirkham B, Henderson DRF, Milazzo SC. Histiocytic necrotizing lymphadenitis in systemic lupus erythematosus Ann Rheum

Dis 1992; 51: 805-7

14. Martínez-Vázquez CA, Bordón J, Rubianes M et al. Enfermedad de Kikuchi. Un estudio clínico patológico de tres casos. An Med Interna (Madrid) 1998; 15: 367-9.

15. Anagnostopoulos I, Hummel M. Korbyuhn P, Papadaki T, Anagnostou D, Stein H. Epstein-Barr virus in Kikuchi-Fujimoto disease. Lancet 1993; 341: 893 (letter).

16. Chan JKC, Wong KC, Mg CS. A fatal case of multicentric Kikuchi's histiocytic lymphadenitis. Cancer 1989; 63: 1856-62.

17. García CE, Girdhar-Gopal Hv, Dorfman DM. Kikuchi-Fujimoto disease of the neck Ann Otol Rhinol Laryngol 1993; 102: 11-15

18. Bataille V, Harland CC, Behrens J et al. Kikuchi disease (histiocytic necrotizing lymphadenitis) in association with HTLV 1. Br J Derm 1997; 136: 610-20.

19. Morillas PJ, González MA, Ferrandis E et al. Enfermedad de KikuchiFujimoto (linfadenitis necrotizante histiocitaria). Acta Otorrinolaring Esp 1996; 47(3): 247-50

20. Kung ITM, Wai-Fu NG, Yuen RWS, Chan HKC. Kikuchi's histiocytic necrotizing lymphadenitis. Diagnosis by fine needle aspiration. Acta Cytologica 1990; 34: 323-8.

21. Nathwani BN. Kikuchi-Fujimoto disease. Am J Surg Pathol 1991; 15: 196-197.

22. Hussein A, Hellquist HB. Necrotizing lymphadenitis of the neck (Kickuchi's disease). APMIS 1994; 102: 633-37.

23. Alvarez R, Sanz MP. Linfadenitis histiocítica necrotizante de KikuchiFujimoto ¿Enfermedad o síndrome? Sangre 1996; 41(5): 337-9.

24. Medeiros LJ, Harvis NL. Lupus lymphadenitis: report of a case with inmunohistologic studies on frozen sections. Hum Pathol 1989; 20: 295-9.

25. Martínez-Vázquez C, Hughes G, Bordón J et al. Histiocytic necrotizing lymphadenitis, Kikuchi-Fujimoto's disease, associated with systemic lupus erythemotosus. Q J Med 1997; 90: 531-33. 